\title{
ARQUEOFAUNAS Y PROCESOS DE INTENSIFICACIÓN EN EL PERÍODO PREHISPÁNICO TARDÍO DE LAS SIERRAS DE CÓRDOBA (ARGENTINA): EL SITIO ARROYO TALAINÍN 2
}

\section{ARCHAEOFAUNAS AND INTENSIFICATION PROCESS DURING THE SIERRAS OF CÓRDOBA LATE PREHISPANIC PERIOD (ARGENTINA): THE ARROYO TALAINÍN 2 SITE}

\author{
Matías E. Medina \\ CONICET-Área de Arqueología, Centro de Estudios Históricos “Prof. Carlos S. A. Segreti”, Miguel C. del Corro \\ 308, Córdoba (5000), E-mail: paleomedina@yahoo.com.ar \\ Presentado el: 05/12/2013 - Aceptado 18/06/2014
}

\section{Resumen}

En este trabajo se presentan los resultados del estudio zooarqueológico realizado sobre los restos de vertebrados recuperados en las excavaciones del sitio prehispánico tardío Arroyo Talainín 2 (Córdoba, Argentina), un abrigo rocoso cuyo talud e interior presentó más de 21.000 restos faunísticos. El conjunto está dominado por cáscaras de huevo de ñandú (Rheidae), con una representación inusualmente densa que se aleja de los patrones regulares observados en otros sitios de la región. Lama sp. y Cervidae ocupan un lugar secundario, con una mayor representación de los elementos axiales, justamente aquellos que son escasos o ausentes en los sitios interpretados como poblados semisedentarios. También presentan en promedio una mayor proporción de partes de alto rendimiento. Sitios como Arroyo Talainin 2 indican que una porción significativa de los recursos explotados durante el Período Prehispánico Tardío -y con frecuencias totalmente distintas- se procesaba, consumía $y$ descartaba recurrentemente en contextos extracomunitarios dispuestos para tal fin, en donde interactuaban numerosas personas y/o grupos fortaleciendo lazos sociales. Se concluye que los cambios observados en el registro arqueológico ca. 1100 AP, más allá de la incorporación de cultígenos y la diversificación de la caza-recolección, también involucraron la intensificación de las relaciones sociales, visible arqueológicamente a partir de la composición de los conjuntos faunísticos y artefactuales. Por último, se destaca la importancia del Número de Especímenes Identificados por taxón (NISP) para cuantificar procesos de intensificación económica a partir de restos óseos altamente fragmentados.

Palabras claves: Período Prehispánico Tardío, Sierras de Córdoba, Zooarqueología, Intensificación 


\begin{abstract}
This paper present the results of the zooarchaeological study carried out on the Late Prehispanic site Arroyo Talainin 2 (Córdoba, Argentina), a rock-shelter with 21000 faunal remains in its deposits. The assemblage is dominated by South-American ostrich eggshells (Rheidae), with an unusually dense representation far from the pattern observed in other regional sites. Lama sp. and Cervidae were secondary exploited on site, showing a high mean utility of skeletal parts and high frequency of axial anatomical elements, those which are scarce or absent in semi-sedentary summer villages. Sites as Arroyo Talainin 2 indicate that a significant portion of the late prehispanic resources included in the subsistence were processed, consumed and discarded regularly in sites associated with large-group food processing and consumption at comunitary level, where social bonds were strengthened. It is concluded that the change observed in the archaeological record ca. $1100 \mathrm{BP}$ with the adoption of a mixed farming and cultivation economy also involved the intensification of social relations, archaeologically visible throught the composition of faunal and artifact assemblages. Finally, the importance of the Number of Identified Specimens per Taxón (NISP) to quantify the economic intensification process in fragmented bones assemblages is highlighted.
\end{abstract}

Keywords: Late Prehispanic Period, Sierras of Córdoba, Zooarchaeology, Intensification

\title{
Introducción
}

Las investigaciones arqueológicas efectuadas en las Sierras de Córdoba (Argentina) han aportado nuevos elementos para una mayor comprensión de la historia prehispánica, en particular del Período Prehispánico Tardío (ca. 1100-360 AP). La información obtenida sugiere que las sociedades tardías desarrollaron una economía mixta que combinaba prácticas agrícolas con aportes significativos de caza-recolección, en donde la flexibilidad fue uno de los rasgos definitorios (Medina et al. 2014). El maíz (Zea mays) y la cerámica fueron extensivamente utilizados, pero en el marco de una estrategia de subsistencia diversificada que incluía un alto grado de movilidad a lo largo del ciclo anual (Medina et al. 2014; Pastor y López 2010; Pastor et al. 2012). De este modo, la caza-recolección continuó cubriendo una parte importante de la dieta, mientras que la agricultura fue estacionalmente interrumpida cuando los recursos silvestres de mayor rendimiento se encontraban disponibles en otros puntos del paisaje. El desarrollo de un nicho económico de amplio espectro, en consecuencia, se acompañó de un grado variable de movilidad residencial, momentos de dispersión/ agregación de los grupos co-residentes y procesos estacionales de abandono de las prácticas productivas para abastecerse de recursos de caza y recolección, con antecedentes que pueden rastrearse al Holoceno Medio.

Dentro del modelo actualmente propuesto, los asentamientos residenciales a cielo abierto o poblados tardíos reflejaban la concentración estival de un conjunto de unidades domésticas para realizar actividades agrícolas, recolectar y capturar pequeños animales, así como para procesar, almacenar y consumir sus productos (Medina 2008; Medina et al. 2008; Medina et al. 2014; Pastor et al. 2012; Pastor et al. 2013;). El grupo co-residente, una vez finalizadas las tareas agrícolas y de recolección, se dispersaba hacia las pampas de altura para capturar ungulados silvestres y mantener la fluidez sociopolítica de la cual dependían. De este modo, los grupos tardíos hicieron un uso estacional y diversificado del paisaje, en donde las unidades domésticas o familiares constituyeron pequeños núcleos relativamente autónomos de producción, consumo y de ocupación del espacio, que se agregaban o dispersaban ajustándose a las cambiantes circunstancias del entorno (Medina et al. 2014; Pastor et al. 2012). 
En este trabajo se presentan los resultados del estudio zooarqueológico realizado sobre los restos de vertebrados recuperados en las excavaciones del sitio prehispánico tardío Arroyo Talainín 2 (Córdoba, Argentina), un abrigo rocoso cuyo amplio talud e interior se caracterizan por densidades inusuales de instrumentos de molienda -fundamentalmente fijos- y residuos vinculados con el consumo de alimentos, con más de 21.000 restos faunísticos. El sitio ofrece una excelente oportunidad para estudiar aquellas ocupaciones que fueron locus de procesamiento y consumo de alimentos pero cuyas características no responden a los poblados residenciales a cielo abierto, intentando determinar su significado en términos de los procesos sociopolíticos y de intensificación económica observados para el Período Prehispánico Tardío. Se plantea que el sitio fue utilizado recurrentemente para procesar y consumir alimentos a escalas supra-domesticas. También se sostiene que el incremento de las actividades colectivas $c a$. 1100 AP fortalecía la integración política entre grupos sociales con relativa fluidez y autonomía, señalando procesos de intensificación en las relaciones sociales.

\section{El sitio}

Arroyo Talainín 2 (AT2) se localiza en el sector occidental del Valle de Salsacate, en las proximidades del faldeo meridional del cerro volcánico Veliz (Figura 1). El entorno inmediato presenta las características ambientales de Bosque Serrano (Luti et al. 1979). El sitio comprende un pequeño abrigo rocoso $\left(16 \mathrm{~m}^{2}\right)$ y un extenso talud con densidades inusuales de restos superficiales, principalmente manos de molinos, desechos e instrumentos líticos, fragmentos cerámicos y una cuenta de collar de piedra (Figura 1). En rocas planas ubicadas en el talud, así como en otra emplazada dentro del abrigo, se destacan más de 80 morteros fijos de dimensiones variables. Se excavaron $8 \mathrm{~m}^{2}$ en distintos sectores del asentamiento, alcanzando profundidades máximas de $90 \mathrm{~cm}$. En su transcurso sólo se reconocieron ocupaciones correspondientes al Período Prehispánico Tardío (ca. 1100-360 AP), sin identificar eventos prolongados de abandono. Con carbón proveniente de una espesa lente ubicada

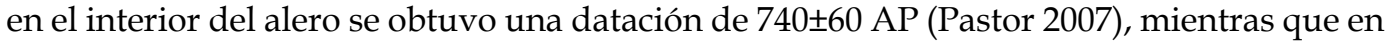
el talud exterior se fecharon dos pisos de ocupación superpuestos en $980 \pm 60$ AP y $900 \pm 50$ $\mathrm{AP}$, señalando los inicios de la ocupación del sitio (Medina et al. 2011a).

Entre los elementos mejor representados en estratigrafía se destacan los restos faunísticos, siendo los camélidos y los huevos de ñandú (Rheidae) los principales recursos consumidos. También se documentó el procesamiento de vegetales, aunque indirectamente -i.e. instrumentos de molienda-, dado que no se conservaron restos arqueobotánicos. La alta proporción de puntas de proyectil líticas y óseas, muchas de ellas con fracturas por uso y/o con indicios de reparación in situ, sugiere que la captura de presas silvestres no fue una actividad secundaria (Medina et al. 2014; Pastor 2007). Entre los escasos recipientes cerámicos reconstruidos $(\mathrm{MNI}=9)$ predominaron los tamaños grandes, comúnmente asociados con la preparación y consumo de volúmenes significativos de comida (Dantas y Figueroa 2008; Pastor 2007). La presencia de instrumentos punzantes de hueso, así como fichas y torteros elaborados sobre tiestos cerámicos, señala que en menor grado también se desarrollaron otras actividades, como el hilado, juegos o procesamiento de pieles (Dantas y Figueroa 2008; Medina et al. 2014; Pastor 2007).

En suma, el registro arqueológico de AT2 señalaría que el sitio se utilizó recurrentemente desde ca. 1000 AP hasta cerca de la llegada de los españoles en el siglo XVI para capturar, procesar y consumir alimentos vegetales y animales, actividad que a juzgar por la cantidad de morteros en superficie, tamaño de los recipientes cerámicos y densidad inusual de los 


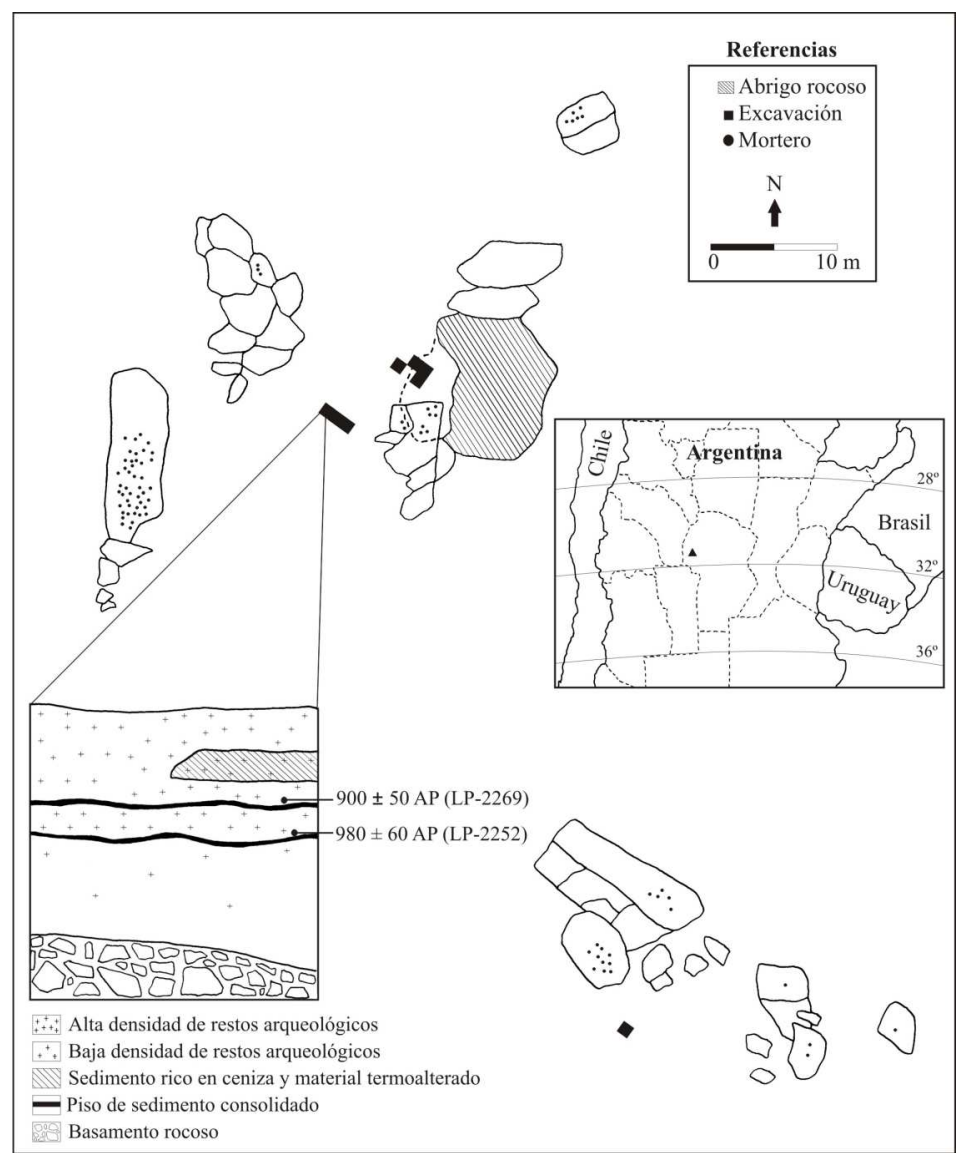

Figura 1: Ubicación geográfica y planta de Arroyo Talainín 2 (Tomado de Medina et al. 2011a).

desechos faunísticos implicó la interacción de numerosas personas y/o grupos sociales. Esto hace que Arroyo Talainín 2 ofrezca una oportunidad única para analizar aspectos económicos y sociopolíticos usualmente invisibles en el registro arqueológico de las sociedades tardías, así como la escala de interacción entre los grupos.

\section{Metodología}

Los especímenes recuperados, tanto en planta como en cernidores de $2 \mathrm{~mm}$ de malla, fueron identificados a nivel de elemento anatómico, porción, lateralidad y al mayor nivel taxonómico posible (Mengoni Goñalons 2010). La abundancia taxonómica relativa se cuantificó mediante el Número de Especímenes Identificado por Taxón (NISP) -también expresado porcentualmente (NISP\%)- y el Número Mínimo de Individuos (MNI; Stiner 1994) ${ }^{1}$. También se consideró el Número Total de Especímenes (NSP) y el Número de Especímenes Indeterminados (NUSP; Lyman 2008).

La diversidad de partes anatómicas de Lama sp. y Ozotoceros bezoarticus fue cuantificada mediante el NISP, la Abundancia Esqueletal Relativa (RSA)² y el Número Mínimo de Elementos (MNE) (Broughton 1999; Grayson y Frey 2004; Mengoni Goñalons 1999). También se estimó el MNE por regiones del esqueleto (Stiner 1994), de manera que los huesos de las 
carcasas se clasificaron en ocho unidades analíticas independientes, cada una reuniendo elementos con ciertas particularidades económicas (cantidad de carne, médula y grasa asociada; De Nigris 2004). Estas partes comprenden: 1) cabeza (cráneo y mandíbula); 2) columna (vértebras cervicales, toráxicas, lumbares y sacro); 3) costillas (costillas y esternón); 4) cinturas (pelvis y escápula);5) extremidades superiores (húmero y fémur); 6) extremidades medias (radio, ulna y tibia); 7) extremidades inferiores (metacarpo y metatarso); 8) calcáneo y pies (calcáneo, falange 1 y falange 2). La frecuencia de las distintas regiones anatómicas fue estandarizada mediante la relación entre el MNE observado con el esperado para cada taxón, procedimiento que facilitó la comparación con otros conjuntos.

Con el mismo objetivo comparativo se examinó la utilidad económica de las partes acarreadas a partir de la utilidad media o retorno promedio por elemento (Mean FUI), cuya fórmula es $\Sigma\left(\mathrm{RSA}_{\mathrm{i}} \times \mathrm{FUI}_{\mathrm{i}}\right) / \Sigma$ RSA (Broughton 1999:59) ${ }^{3}$. De esta manera, conjuntos con valores altos de utilidad media sugerirían, en términos relativos, una mayor representación de partes de alto rendimiento económico y viceversa.

La historia tafonómica fue reconstruida considerando las frecuencias de huellas culturales y marcas naturales a partir del NISP (Stiner 1994). La identificación de las mismas fue realizada macroscópicamente siguiendo las indicaciones de Blumenschine et al. (1996) y Stiner (2005). La extensión de la fragmentación se cuantificó mediante la relación entre el NISP y el NSP (NISP/NSP) sin considerar los fragmentos de cáscaras de huevo (Wolverton et al. 2011). Por otro lado, el porcentaje de falanges 1 y 2 completas -elementos de escaso contenido de médula-, así como el índice de fragmentación de Lama sp. y O. bezoarticus (NISP/MNE), se utilizan para indagar cuan intensivo fue el aprovechamiento de la médula de ungulados (Wolverton et al. 2011).

La integridad de las muestras de Lama sp. y O. bezoarticus también fue evaluada en términos de conservación diferencial de los distintos elementos, estableciéndose correlaciones entre el RSA y la densidad ósea a partir de datos de Elkin (1995). Finalmente, la meteorización de los huesos de Lama sp. fue analizada de acuerdo a la escala ordinal propuesta por Behrensmeyer (1978), con la intención de discutir la duración, intensidad y continuidad en el uso del sitio.

\section{Resultados}

En AT2 se recuperó uno de los principales conjuntos arqueofaunísticos del período prehispánico tardío de las Sierras de Córdoba (NSP=21.281), superando por varias veces el tamaño de los conjuntos obtenidos en sitios residenciales con mayor superficie intervenida (Figura 2). Cerca de la mitad del conjunto fue clasificada como no-identificable, aún cuando la fragmentación no difiere de la del resto de los conjuntos tardíos (Tabla 1) (Medina y Pastor 2012). Dentro de los especímenes que pudieron ser determinados taxonómicamente (NISP=10.941), más de la mitad se correspondieron a cáscaras de huevo de Rheidae (NISP= $6029 ; \mathrm{NISP} \%=55,10, \mathrm{MNI}=12$ ), indicando que fue el principal recurso animal consumido en el sitio (Figura 3). Sin embargo, el MNI podría estar subestimado frente a los altos valores de NISP, que a escalas arqueológicas asume que cada espécimen se corresponde con un individuo (Lyman 2008). También porque el MNI es presentado sin diferenciar por capas ni cuadrículas -que no siempre fueron contiguas (Figura 1)-, factor al que el MNI es sensible (Lyman 2008). 


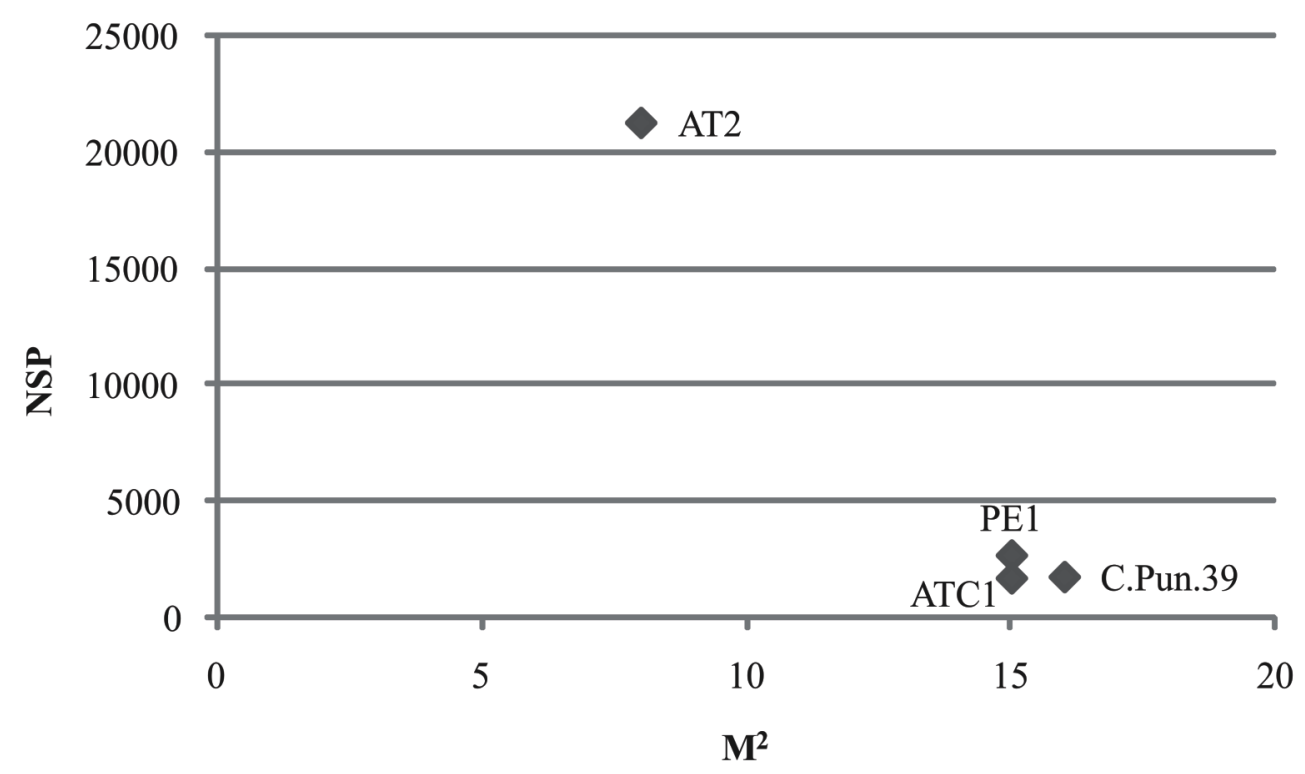

Figura 2. Número Total de Especímenes (NSP) y superficies intervenidas $\left(\mathrm{m}^{2}\right)$.

\begin{tabular}{|c|c|c|c|c|c|c|c|}
\hline Taxón & MNI & NISP & NISP he & NISP fract & NISP a & NISP ro & NISP car \\
\hline Lama sp. & 6 & 701 & 59 & 47 & 264 & 5 & 1 \\
\hline Mazama sp. & 1 & 14 & 3 & --- & 5 & --- & --- \\
\hline Odocoleinae cf. Mazama sp. & 1 & 3 & 1 & --- & 2 & --- & --- \\
\hline O. bezoarticus & 2 & 73 & 15 & 7 & 13 & --- & --- \\
\hline Odocoleinae cf. O. bezoarticus & & 9 & --- & --- & 3 & --- & --- \\
\hline Odocoleinae & 2 & 165 & 12 & 6 & 61 & --- & --- \\
\hline P. taiacu & 1 & 1 & --- & --- & 1 & --- & --- \\
\hline Artiodactyla & --- & 222 & 13 & --- & 77 & --- & --- \\
\hline Euphractinae & 1 & 224 & --- & --- & 68 & $\begin{array}{ll}-- \\
\end{array}$ & --- \\
\hline Dasvpodidae & 2 & 22 & 2 & --- & 4 & --- & --- \\
\hline Dolichotis patagonum & 1 & 3 & 1 & --- & 1 & --- & --- \\
\hline Caviinae & 1 & 2 & --- & --- & --- & --- & --- \\
\hline Ctenomus sp. & 1 & 33 & --- & --- & --- & --- & 1 \\
\hline Rodentia & 1 & 2 & --- & --- & --- & --- & 1 \\
\hline Mammalia (tamaño pequeño) & --- & 131 & 4 & --- & 75 & --- & --- \\
\hline Mammalia (tamaño med-grande) & $\begin{array}{ll}-- \\
\end{array}$ & 3220 & 247 & 189 & 1369 & --- & 4 \\
\hline Pristidactulus sp. & 1 & 21 & --- & --- & --- & --- & --- \\
\hline Tupinambis sp. & 1 & 4 & 1 & --- & 2 & --- & --- \\
\hline Squamata cf. Teiidae & 1 & 1 & --- & --- & --- & --- & --- \\
\hline Rheidae & 1 & 6 & 2 & --- & 2 & --- & --- \\
\hline Rheidae (huevos) & 12 & 6029 & --- & --- & 5562 & --- & --- \\
\hline Tinamidae & 2 & 9 & 1 & --- & --- & --- & $\begin{array}{c}-- \\
\end{array}$ \\
\hline Ave indet & 1 & 4 & -- & --- & 1 & --- & --- \\
\hline NUSP & --- & 10340 & 391 & 340 & 3225 & 1 & 2 \\
\hline NSP & --- & 21281 & 752 & 589 & 10735 & 6 & 9 \\
\hline
\end{tabular}

Tabla 1. Número Mínimo de Individuos (MNI) y Número de Especímenes Identificados por

Taxón (NISP) de Arroyo Talainín 2. NISP hc: NISP con huellas de corte; NISP fract: NISP con fractura antrópicas; NISP q: NISP quemado; NISP ro: NISP con marcas de roedores; NISP car: con marcas de dientes de carnívoros o humanos. 


\section{NISP\% $\%$}

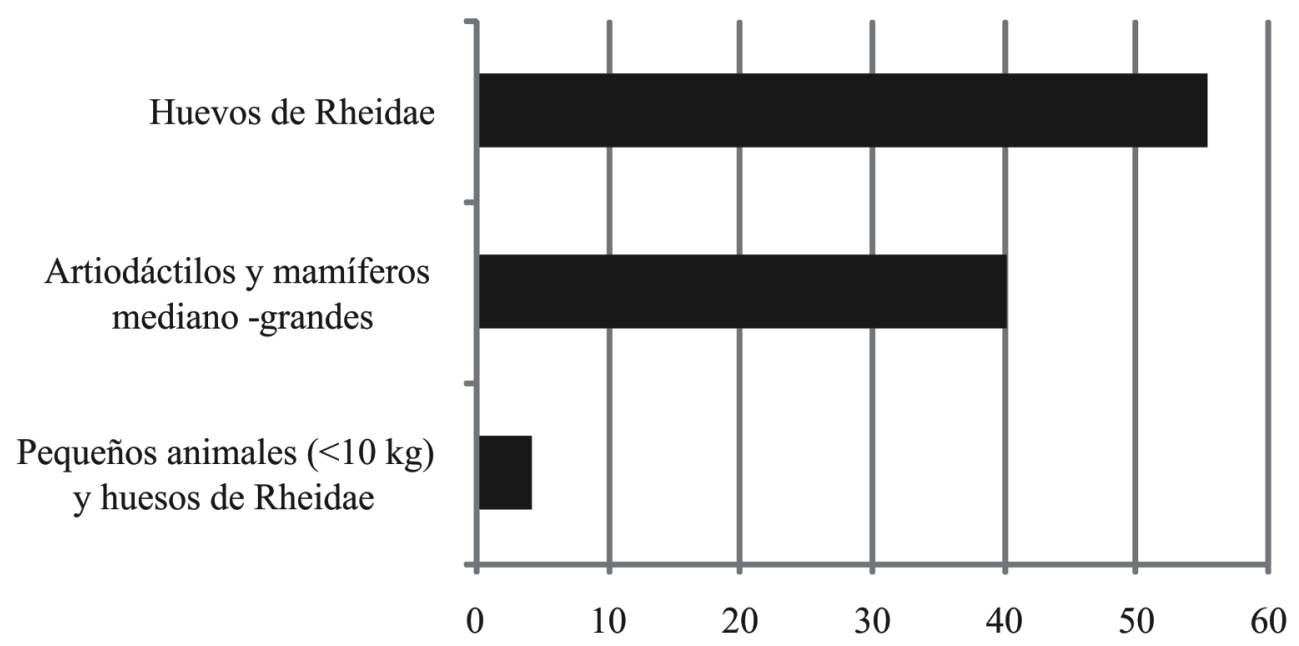

Figura 3. NISP\% de los huevos de Rheidae, Artiodáctilos/mamíferos mediano-grandes y pequeños animales $(<10 \mathrm{~kg}$ ) del sitio arqueológico Arroyo Talainín 2.

Las especies Rhea cf. $R$ americana $(\mathrm{NISP}=19)$ y Rhea cf. $R$. pennata $(\mathrm{NISP}=32)$ fueron identificadas a partir del estudio de la densidad de poros en los fragmentos de cáscaras (Medina et al. 2011a). Los especímenes que no reunieron los criterios adecuados para este tipo de análisis -i.e. presentaban tamaños de 10-20 mm de dimensión máxima, desgaste o estaban termoalterados- fueron asignados a Rhea spp. (NISP=5978). R. americana actualmente habita espacios abiertos al NW y SW de la provincia, por lo que sus huevos pudieron ser obtenidos dentro del radio de aprovisionamiento del sitio, posiblemente en la Pampa de Pocho, con espacios abiertos favorables para estas aves no-voladoras. En cambio, R. pennata no ha sido citada para Córdoba. Las referencias más cercanas se corresponden a la subespecie R. pennata garleppi en las tierras altas del noroeste de San Juan y La Rioja, mientras que R.p. pennata habita el norte de Río Negro, sur de Mendoza, sur de Buenos Aires y suroeste de La Pampa. La presencia de este taxón podría estar indicando que durante el Período Prehispánico Tardío el paisaje de las Sierras de Córdoba presentaba una mayor biodiversidad para la cual el ambiente actual no es un buen análogo, con R. pennata en las pampas de altura o en las cercanías del sitio (Medina et al. 2011b).

Es difícil de establecer en base a atributos tafonómicos confiables si los fragmentos de huevo de AT2 son producto de nidadas fósiles, descarte del consumo antrópico y/o consecuencia de la rotura de huevos utilizados como contenedores. Sin embargo, el no esperar nidos a escasos metros de reparos rocosos (Barri et al. 2009), la densidad inusual de restos, su asociación con artefactos líticos, cerámicos y óseos, la identificación de dos especies del género Rhea, junto con el altísimo porcentaje de especímenes quemados (NISP\%=92,25) -más del doble respecto al resto de los vertebrados-, indican una asociación consistente entre el recurso y la ocupación humana del sitio. La ausencia de perforaciones o motivos gravados en los huevos reduce la posibilidad de su uso como contenedores, artefactos relativamente comunes en culturas etnográficas y arqueológicas (Fiore y Borella 2010; Stiner 2005; Texier 
et al. 2010). En pocas palabras, todo indica que AT2 se ocupó recurrentemente en primavera, coincidiendo con la disponibilidad de huevos, siendo los mismos transportados desde distancias variables hacia el asentamiento y cocinados junto a fogones (Medina et al. 2011a).

Los ungulados, cuyos restos derivaron de la importación de partes anatómicas desde ambientes de pastizal abierto, ocupan un lugar secundario en cuanto NISP y MNI, aun duplicando los valores obtenidos en otros sitios. Sin embargo, el aporte como carcasas individuales fue importante en comparación con los huevos. Más aun cuando existen argumentos teóricos y metodológicos para asumir que cada espécimen se depositó en forma independiente del resto a excepción de que estén articulados, restando valor analítico al MNI. En este sentido, no hay una técnica válida para determinar que, por ejemplo, un fémur izquierdo se corresponda al mismo individuo que un fémur derecho (Bovy 2012), sobre todo en conjuntos donde los fechados radiocarbónicos promedian décadas o siglos y las unidades de excavación están espacialmente discontinuas. Además, el MNI se construye en función del NISP, siendo medidas redundantes (Grayson y Frey 2004).

La presencia de modificaciones de indiscutible origen antrópico, como huellas de corte y percusión -además de especímenes termoalterados-, permiten sostener que Camelidae y Cervidae se incorporaron al conjunto como resultado de actividades de procesamiento, cocción y consumo (Tabla 1). El bajo porcentaje de falanges enteras y la relación NISP/MNE en Lama sp., por otro lado, sugiere el aprovechamiento intensivo de las carcasas de Camelidae (Munro y Bar Oz 2005), en particular frente a la menor fragmentación de Cervidae (Tabla 2).

\begin{tabular}{|c|c|}
\hline Variables & AT2 \\
\hline NSP:NISP & 3,10 \\
\hline $\begin{array}{l}\text { Mediana NISP:MNE de } \\
\text { Camelidae }\end{array}$ & 2,855 \\
\hline $\begin{array}{l}\text { Mediana NISP:MNE de O. } \\
\text { bezoarticus }\end{array}$ & 1 \\
\hline $\begin{array}{l}\text { \% Falanges } 1 \text { y } 2 \text { de Camelidae } \\
\text { completas* }\end{array}$ & $7,47 \%$ \\
\hline $\begin{array}{l}\text { \% Falanges } 1 \text { y } 2 \text { de } O \text {. bezoarticus } \\
\text { completas* }\end{array}$ & $50 \%$ \\
\hline $\begin{array}{l}\text { \%bone-based NISP de Camelidae } \\
\text { con huellas de corte }\end{array}$ & $\begin{array}{c}9,93 \% \\
(\mathrm{NISP}=59)\end{array}$ \\
\hline $\begin{array}{l}\text { \%bone-based NISP de } O \text {. } \\
\text { bezoarticus con huellas de corte }\end{array}$ & $\begin{array}{c}19,73 \% \\
(\mathrm{NISP}=15)\end{array}$ \\
\hline $\begin{array}{l}\text { \%NISP de Camelidae } \\
\text { termoalterado }\end{array}$ & $\begin{array}{c}37,66 \% \\
(\mathrm{NISP}=264)\end{array}$ \\
\hline $\begin{array}{l}\text { \%NISP de O. bezoarticus } \\
\text { termoalterado }\end{array}$ & $\begin{array}{c}19,51 \% \\
(\mathrm{NISP}=16)\end{array}$ \\
\hline $\begin{array}{l}\text { \%bone-based NISP de Camelidae } \\
\text { con fractura antrópica }\end{array}$ & $\begin{array}{c}7,91 \% \\
(\mathrm{NISP}=47)\end{array}$ \\
\hline $\begin{array}{l}\text { \%bone-based NISP de } O \text {. } \\
\text { bezoarticus con fractura antrópica }\end{array}$ & $\begin{array}{c}7,89 \% \\
(\mathrm{NISP}=6)\end{array}$ \\
\hline
\end{tabular}

Tabla 2.Principales variables para indagar la extensión y la intensidad de la fragmentación en el sitio Arroyo Talainín 2. *No se contabilizan los especímenes que sólo son epífisis proximales sin fusionar. 


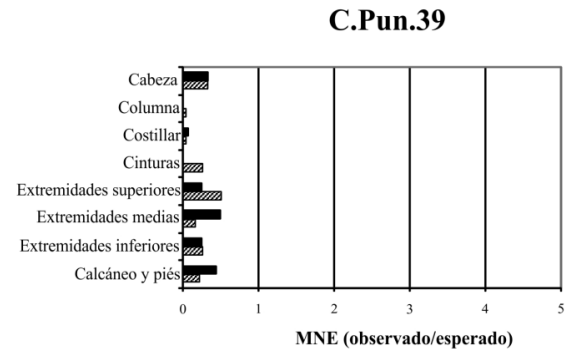

Arroyo Tala Cañada 1

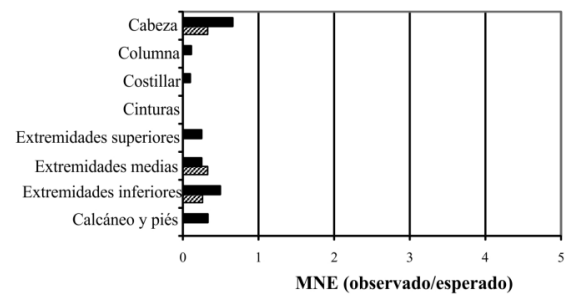

Puesto La Esquina 1

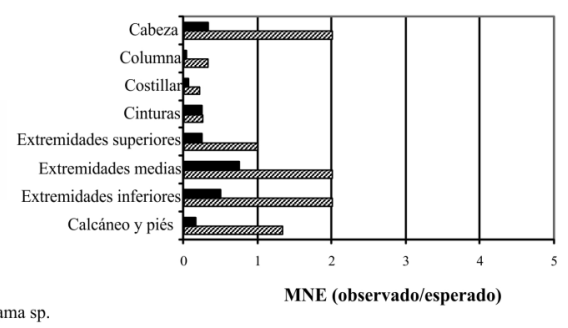

\$O. bezoarticus

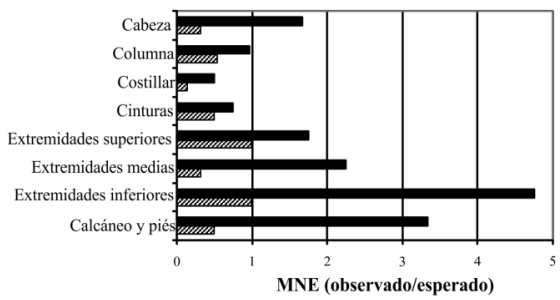

Figura 4. Patrones de representación anatómica de Lama sp. y O. bezoarticus en AT2 respecto a otros sitios.

Lamasp. $(80 \mathrm{~kg})+$ O. bezoarticus $(35 \mathrm{~kg})$

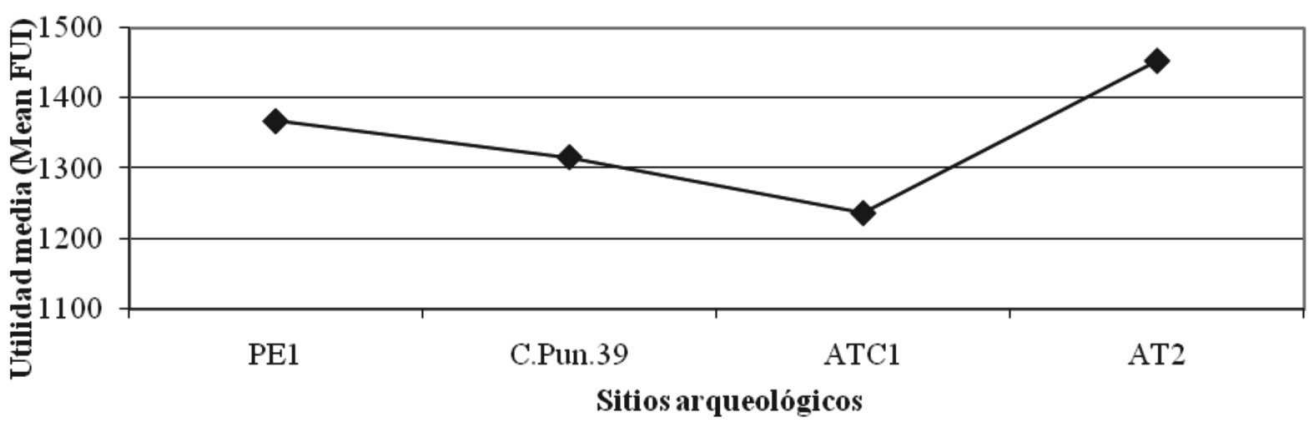

Figura 5. Utilidad media o retorno promedio por elemento de Lama sp. y O. bezoarticus en AT2 respecto a otros sitios.

Un aspecto a destacar en el conjunto de Lama sp. y O. bezoarticus de AT2 es la mayor representación de elementos axiales ricos en contenido cárnico -columna y costillares-. Se trata justamente de aquellas partes que tienen mayores posibilidades de deshidratación para el consumo diferido (De Nigris 2004) y que son escasas o ausentes en los sitios aldeanos, reforzando la idea de que estos últimos no reflejan la totalidad de los recursos consumidos (Figura 4). También presentan en promedio una mayor proporción de partes de alto rendimiento, indicando que hacia el sitio se estarían transportando desde las pampas de altura -hábitat de guanacos y venados- partes anatómicas con alto contenido de carne asociada al hueso (Figura 5). Este aspecto no es tan claro para los sitios aldeanos, incluso en 
aquellos con acceso inmediato a los recursos de los sectores serranos de altura, en donde el transporte selectivo en función de la anatomía económica no fue un factor significativo en la formación de los depósitos (Figura 4 y 5).

Aun cuando el perfil anatómico de Lama sp. muestra una relación moderada con la densidad ósea (Tabla 3), otros aspectos del conjunto faunístico desestiman la existencia de sesgos post-depositacionales. En primer lugar, el patrón anatómico de O. bezoarticus no se relaciona con la densidad ósea, restando importancia estadística a la correlación moderada de Lama sp. y sugiriendo historias tafonómicas independientes, tal como lo evidencia la fragmentación diferencial. Por otro lado, la meteorización de Lama sp. está dominada casi en forma absoluta por el estadio 2 (Figura 6), restando importancia a la destrucción in situ de los restos e indicando eventos de ocupación cortos pero repetidos en el tiempo. El resto del conjunto también presenta un buen estado de conservación, incluyendo la preservación excepcional de ca. 6.000 fragmentos de cáscaras de huevos (Medina et al. 2011a) y abundantes valvas de gastrópodos terrestres -i.e. Epiphragmophora sp., Plagiodontes sp., etc.- (Pastor 2007), todos ellos de baja densidad relativa y escaso potencial de conservación (Bovy 2012). Además, AT2 tiene el conjunto tardío de Lama sp. más importante en términos cuantitativos, duplicando el de otros sitios que justamente no presentan covariación con esta variable (Medina y Pastor 2012). Finalmente, la elevada frecuencia de huellas de procesamiento y consumo, junto con fracturas de carácter intencional y termoalteraciones, indican un proceso de destrucción pre-depositacional de Lama sp. que claramente responde al uso intensivo de la carcasas, incluyendo la extracción de médula y grasa, en un contexto de intensificación económica (sensu Munro y Bar Oz 2005).

\begin{tabular}{|l|c|c|}
\hline \multirow{2}{*}{ Taxón } & \multicolumn{2}{|c|}{ Densidad ósea } \\
\cline { 2 - 3 } & Correlación & $\begin{array}{c}\text { Puntos } \\
\text { ploteados }\end{array}$ \\
\hline Lama sp. & $\begin{array}{r}\mathrm{r}_{\mathrm{s}}=.50211 \\
\mathrm{p}=0,02037\end{array}$ & 21 \\
\hline O. bezoarticus & $\begin{array}{c}\mathrm{r}_{\mathrm{s}}=-0,06651 \\
\mathrm{p}=0,80664\end{array}$ & 16 \\
\hline
\end{tabular}

Tabla 3. Correlaciones estadísticas utilizando el test de Spearman entre RSA y la densidad ósea (Elkin 1991).

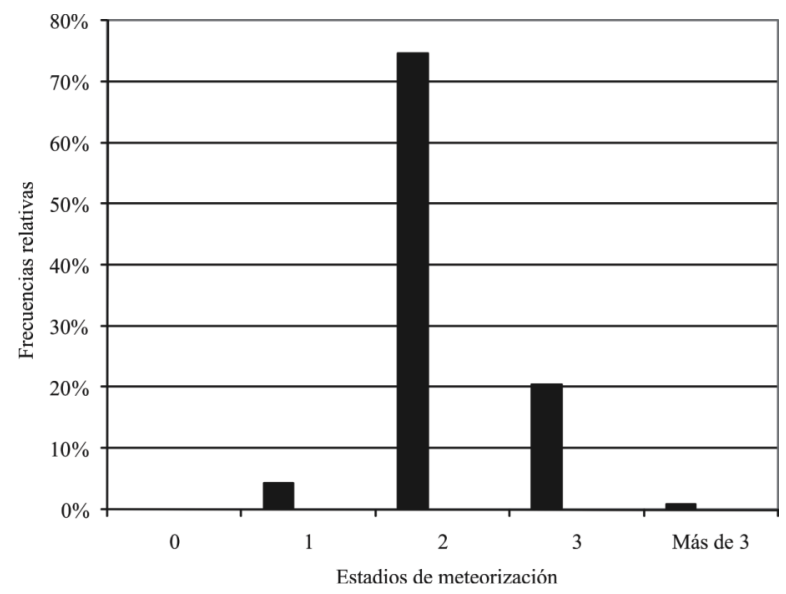

Figura 6. Perfil de meteorización de Lama sp. provenientes de Arroyo Talainin 2 (N:236) 
El resto de los taxones, fundamentalmente pequeños animales, en su mayoría no presenta huellas de origen antrópico y posiblemente su depositación responda a causas naturales. Un ejemplo de esto son los roedores caviomorfos Ctenomys sp. y Caviinae, sin huellas de corte y/o termoalteraciones, trazas que son comunes en otros sitios de la región (Medina et al. 2012; Medina y Pastor 2012). Una excepción la conforman los armadillos Euphractinae, representados por osteodermos asignados a Chaetophractus villosus y Ch. vellerosus, con una alta frecuencia de especímenes afectados por la acción del fuego que sugiere que fueron transportados hacia el sitio y cocinados junto a fogones (Soilbenzon et al. 2013).

\section{Discusión}

Las cáscaras de huevo de AT2, más allá de su fragmentación extensiva, poseen una representación inusualmente densa que se aleja de los patrones regulares observados en otros sitios de la región, incluyendo del mismo valle de Salsacate -i.e. Arroyo Tala Cañada 1 (Medina y Pastor 2012)-. Esto sugiere que la obtención, procesamiento y consumo de este recurso, junto con partes del esqueleto de Lama sp. ricos en carne y recursos vegetales como la algarroba (Prosopis spp.) y/o maíz, fueron las principales actividades realizadas en el sitio. La concentración de restos faunísticos y el número significativo de puntas de proyectil, junto con las evidencias asociadas a preparar y servir alimentos, sugiere que estas tareas se realizaban a una escala supra-doméstica, implicando la interacción de individuos provenientes de poblados localizados en los alrededores o bien de zonas más alejadas, tal como lo evidencia la presencia de elementos faunísticos de las pampas de altura ca. $30 \mathrm{~km}$ del alero. La meteorización de Lama sp., en combinación con las dataciones radiocarbónicas, es indicativa de que las ocupaciones fueron breves, con grandes eventos de descarte, pero indiferenciables estratigráficamente.

Otros sitios tardíos de la porción meridional del valle de Guasapampa, ca. $25 \mathrm{~km}$ al norte de AT2, también presentaron alto NISP\% asignado a huevos de Rheidae (Tabla 4). Se trata, fundamentalmente, de ocupaciones en aleros de carácter doméstico vinculadas con la dispersión estacional de los grupos co-residenciales. Sin embargo, fueron obtenidos a partir de la intervención de pequeñas superficies, siendo los restos óseos recuperados escasos y fragmentados por mecanismos post-depositacionales, lo que puede haber sobredimensionado la abundancia relativa de las cáscaras (Lyman 2008; Marshall y Pilgram 1993). La comparación del NISP\% con los bajos valores de MNI soportan esta hipótesis (Medina et al. 2011a).

En los sitios agrícolas-residenciales a cielo abierto, considerados los principales locus de actividades y unidades fundamentales del patrón de asentamiento, el NISP y el MNI de huevos siempre fue marginal, independientemente de la superficie intervenida (Medina y Pastor 2012; Medina et al. 2011a). Incluso en aquellos que tuvieron acceso inmediato a los ambientes de pastizal -hábitat potencial de estas aves corredoras-, como Puesto La Esquina 1 (Pampa de Olaen), los fragmentos de cáscaras apenas superan el $4 \%$ del NISP (Medina y Pastor 2012; Medina et al. 2011a). Esto llevó a considerar que los huevos de Rheidae fueron recursos de uso ocasional o complementario, sin discutir en profundidad su significado y/o rol en el contexto de cada uno de los sitios. Este panorama es extensible a distintas ocupaciones tardías de la Pampa de Achala (Tabla 4), en donde los camélidos dominan taxonómicamente los conjuntos casi en forma absoluta, seguido por cérvidos y pequeños mamíferos. 


\begin{tabular}{|c|c|c|c|c|c|c|}
\hline Sitio & Emplazamiento & Localización & Cronología & NISP $\%$ & MNI & Fuente \\
\hline Potrero de Garay & cielo abierto & $\begin{array}{l}\text { Valle de Los } \\
\text { Reartes }\end{array}$ & $310 \pm 75 \mathrm{AP}$ & $1,59 \%$ & 1 & Medina (2002) \\
\hline Puesto La Esquina 1* & cielo abierto & Pampa de Olaen & $\begin{array}{l}365 \pm 38 \text { AP } \\
362 \pm 43 \text { AP }\end{array}$ & $4,11 \%$ & 1 & Medina (2009) \\
\hline C.Pun.39* & cielo abierto & Valle de Punilla & $\begin{array}{l}525 \pm 36 \mathrm{AP} \\
716 \pm 39 \mathrm{AP} \\
854 \pm 39 \mathrm{AP}\end{array}$ & $0,36 \%$ & 1 & Medina (2009) \\
\hline Arroyo Tala Cañada $1^{*}$ & cielo abierto & Valle de Salsacate & $\begin{array}{c}900 \pm 70 \text { AP } \\
1028 \pm 40 \text { AP }\end{array}$ & $2,48 \%$ & 1 & $\begin{array}{c}\text { Pastor y } \\
\text { Berberián (2007) }\end{array}$ \\
\hline Los Algarrobos $1^{*}$ & cielo abierto & Pampa de Olaen & $949 \pm 40 \mathrm{AP}$ & $0 \%$ & 1 & Medina (2008) \\
\hline Arroyo Talainín 2 & abrigo rocoso & Valle de Salsacate & $\begin{array}{l}740 \pm 60 \mathrm{AP} \\
980 \pm 60 \mathrm{AP} \\
900 \pm 50 \mathrm{AP}\end{array}$ & $55,31 \%$ & 12 & Este trabajo \\
\hline Río Yuspe 11 & abrigo rocoso & Pampa de Achala & $\begin{array}{l}1540 \pm 50 \mathrm{AP} \\
1170 \pm 50 \mathrm{AP}\end{array}$ & $0,96 \%$ & 1 & Pastor (2007) \\
\hline Río Yuspe 14 & abrigo rocoso & Pampa de Achala & $640 \pm 70 \mathrm{AP}$ & $4,42 \%$ & 1 & $\begin{array}{l}\text { Rivero et al. } \\
\text { (2010) }\end{array}$ \\
\hline Achalita 9 & abrigo rocoso & $\begin{array}{c}\text { Valle de } \\
\text { Traslasierra }\end{array}$ & $\mathrm{s} / \mathrm{d}$ & $42,24 \% * *$ & 1 & $\begin{array}{l}\text { Rivero et al. } \\
\text { (2010) }\end{array}$ \\
\hline Yaco Pampa 1 & cielo abierto & Guasapampa & $1360 \pm 60 \mathrm{AP}$ & $23,92 \%$ ** & 1 & $\begin{array}{l}\text { Rivero et al. } \\
\text { (2010) }\end{array}$ \\
\hline Cerco de la Cueva 3 & abrigo rocoso & Guasapampa & $390 \pm 60 \mathrm{AP}$ & $84,01 \%$ ** & 1 & $\begin{array}{c}\text { Rivero et al. } \\
(2010)\end{array}$ \\
\hline $\begin{array}{l}\text { Cerco de la Cueva } \\
\text { Pintada }\end{array}$ & abrigo rocoso & Guasapampa & $\mathrm{s} / \mathrm{d}$ & $68,44 \% * *$ & 1 & $\begin{array}{l}\text { Rivero et al. } \\
\text { (2010) }\end{array}$ \\
\hline Charquina 2 & abrigo rocoso & Guasapampa & $\begin{array}{l}1060 \pm 60 \mathrm{AP} \\
1190 \pm 70 \mathrm{AP}\end{array}$ & $38,75 \%$ & 1 & $\begin{array}{l}\text { Rivero et al. } \\
\text { (2010) }\end{array}$ \\
\hline
\end{tabular}

Tabla 4. Porcentaje del Número de Especimenes Identificados (NISP\%) y Número Mínimo de Individuos (MNI) de huevos de Rheidae en sitios prehispánicos tardíos de las Sierras de Córdoba.

*: poblados tardíos;**: El cálculo de porcentaje es cuestionable como consecuencia del pequeño tamaño de la muestras.

El patrón observado no parece responder a sesgos causados por la conservación diferencial, errores de muestreo o al empleo de distintas técnicas de recuperación, dado que los fragmentos -incluyendo algunos de escasos milímetros, coloración homogénea y buen estado de conservación-, en mayor o menor medida están presentes en los sitios. Además, los restos de ungulados asociados no presentan preservación mediatizada por la densidad ósea, evidenciando una baja descomposición in situ de las cáscaras más allá de la fragmentación (Medina y Pastor 2012). Una de las explicaciones posibles puede estar asociada con la estacionalidad de las ocupaciones, de modo que los sitios no eran ocupados cuando las nidadas estaban disponibles en los alrededores. Sin embargo, la hipótesis sólo es válida para argumentar su ausencia en sitios de los sectores serranos de altura, ambiente que parece haber sido ocupado fundamentalmente en invierno, cuando los grupos co-residenciales finalizaban las tareas agrícolas y se dispersaban para capturar ungulados silvestres. En cambio, no explica satisfactoriamente la situación de los poblados tardíos cuya ocupación, al presentar evidencias de producción agrícola -instrumentos, semillas, surcos, etc- y restos óseos de Tupinambis sp., necesariamente coincidió con la disponibilidad del recurso (Medina et al. 2014).

El paisaje faunístico regional sugiere, entonces, que el consumo de huevos se realizaba en sitios claramente disociados de los espacios residenciales, ya sea en pequeñas ocupaciones domésticas en Guasapampa o a gran escala en sitios de propósitos especiales asociados al procesamiento de volúmenes significativos de alimentos. Entre estos últimos 
puede ubicarse a AT2, en donde junto con los huevos también se transportaron, procesaron y consumieron en forma comunitaria partes anatómicas de ungulados silvestres con alta utilidad económica.

Los artefactos y técnicas utilizados en AT2 para la preparación de la comida -molienda, instrumentos de corte, fragmentación de los huesos, hervido y asado- no difieren de aquellas empleadas en los contextos domésticos (Dantas y Figueroa 2008; Medina 2010; Medina y Pastor 2012; Pastor y Berberián 2007). La evidencia, de esta manera, permite argumentar que los eventos comunitarios de preparación y consumo de alimentos fueron extensiones de las actividades domésticas pero a escalas de interacción más inclusivas, en donde también se buscó extraer la mayor cantidad de nutrientes de los recursos procesando intensivamente las carcasas. Este aspecto es observable en los valores de NISP y en la baja proporción de falanges de Lama sp. enteras, indicativo de que, aun su reducido tamaño, esta fuente de médula fue intensamente utilizada. La ausencia de recipientes asociados al almacenaje de líquidos y sólidos, comunes en los contextos residenciales (Dantas y Figueroa 2008; Medina 2010), señala que el procesamiento de recursos almacenados no fue importante en AT2, siendo consistente con ocupaciones cortas que coincidían con la disponibilidad de huevos en los alrededores y su consumo en forma inmediata. La alta fragmentación de los restos faunísticos y la identificación de ollas de tamaño grande llevan a hipotetizar que los ungulados ingresados al sitio fueron segmentados y preparados en guisos junto con vegetales silvestres. La grasa ósea también pudo ser explotada agregando fragmentos de epífisis o tejido trabecular al caldo, que en el registro zooarqueológico están representados en las categorías Mammalia sp. indet (tamaño mediano-grande) y NUSP. Esta forma de cocción, en contraposición con el posible asado de los huevos, permitió repartir porciones relativamente equitativas de comida entre los niños, mujeres y hombres, así como entre las unidades domésticas y linajes que participaban del evento, denotando relaciones igualitarias entre los actores sociales (Potter y Ortman 2004).

La existencia de sitios que manifiestan eventos supra-domésticos cobra sentido al tener en cuenta los cambios económicos y sociales observados en el paisaje arqueológico de fines del Holoceno Tardío. Entre los principales cambios, se destaca la incorporación de prácticas agrícolas a pequeña escala, el incremento de la importancia de la caza-recolección y un crecimiento demográfico significativo en un territorio circunscripto por poblaciones vecinas en expansión (Medina et al. 2014; Pastor y López 2010; Pastor et al. 2012). Estas circunstancias derivaron en la creación de nuevas tecnologías, la reducción de los rangos de movilidad, el aumento de la violencia interpersonal y la ocupación de todos los espacios serranos (Pastor et al. 2012). La intensificación de las relaciones sociales, expresada arqueológicamente en el consumo colectivo de recursos silvestres, jugó entonces un papel importantísimo al fortalecer los lazos comunitarios entre los grupos tardíos, manteniendo la fluidez sociopolítica para establecer alianzas, reducir conflictos, intercambiar información y resolver incongruencias en la distribución espacial de recursos esenciales para la subsistencia.

En tal sentido, el contexto no-habitacional documentado en AT2, en particular su conjunto faunístico, ofrece una excelente oportunidad para discutir arqueológicamente como los procesos económicos y sociales estuvieron indisolublemente unidos en su ejecución, siendo históricamente delimitados solo por razones metodológicas y/o operativas (Bonomo 2006). En consecuencia, un análisis zooarqueológico restringido a ponderar la importancia de los huevos de ñandú y/o de los artiodáctilos en la subsistencia tardía sería limitativo, dado que los contextos asociados a su consumo también fueron fundamentales para la reproducción 
social de los grupos prehispánicos. En este sentido, las actividades colectivas realizadas en AT2 facilitaron el acceso a ítems heterogéneamente distribuidos en el paisaje pero necesarios para la subsistencia, como huevos, rocas y ungulados. Al mismo tiempo, cementaban vínculos de identidad entre pequeños grupos dispersos, en un contexto de intensificación económica y de complejización de las relaciones sociales.

\section{Conclusiones}

El estudio arqueológico del Período Prehispánico Tardío de las Sierras de Córdoba durante décadas asumió que la incorporación de prácticas agrícolas rápidamente derivó en una mayor dependencia de los cultivos -principalmente maíz-y en la sedentarización de los grupos en poblados compuestos por viviendas semi-subterraneas o casas-pozos (Berberián 1984; Canals Frau 1953; González 1943; Laguens y Bonnin 2009; Outes 1911; Serrano 1945). Sin embargo, la ausencia de arquitectura permanente y la baja inversión en infraestructura agrícola, así como el resto de la evidencia contextual, impiden sostener que la agricultura haya sido la estrategia económica de mayor jerarquía y justificar un sedentarismo estricto (Medina et al. 2014). En cambio, la evidencia sugiere patrones de subsistencia y movilidad flexibles, con unidades domésticas o familiares relativamente autónomas que ajustaban la localización de su asentamiento y el tamaño de los grupos de acuerdo a las cambiantes circunstancias del entorno.

Arroyo Talainín 2 es una pieza clave en esta discusión. La disociación de los sitios como AT2 de los grandes asentamientos residenciales ocupados en primavera-verano corrobora que estos últimos no concentraban la totalidad de los recursos consumidos. En este sentido, AT2 indica que una porción de los mismos -y con frecuencias totalmente distintas- se procesaba, consumía y descartaba fuera del ámbito de los poblados, en localizaciones socialmente dispuestas para tal fin (Pastor 2007; Medina y Pastor 2012; Medina et al. 2011a). La abundancia de restos óseos asignados a Lama sp. y O. bezoarticus es indicativa de que las reuniones en AT2 no necesariamente dependían de la disponibilidad local de huevos. Una porción significativa de los alimentos consumidos, en particular elementos anatómicos de ungulados silvestres ricos en carne, se transportaban desde distancias considerables. La apropiación, procesamiento y consumo colectivo de recursos reflejaría, en consecuencia, acciones no solo de importancia económica asociada al acceso a proteína animal, sino que también estarían involucrados procesos de identidad, afiliación y probablemente de diferenciación social entre las unidades sociales participantes.

En segundo lugar, la abundancia de puntas de proyectil, cáscaras de huevos y restos óseos de ungulados sugiere que, aun cuando los cultivos fueron importantes en términos económicos, la caza-recolección continuó ocupando un lugar significativo dentro de la subsistencia tardía. Los argumentos se robustecen al considerar el número de instrumentos de molienda y la localización del sitio junto a grandes algarrobales, aun cuando el procesamiento de maíz no puede ser descartado. De este modo, los datos indican que las sociedades tardías definían su rango de acción en torno a poblados agrícolas ocupados durante la estación productiva y sitios dispersos en el paisaje serrano vinculados a la realización de actividades extractivas. El resto de la información contextual, incluyendo estudios distribucionales, bioarqueológicos, zooarqueológicos, arqueobotánicos y de la organización de la tecnología, también apuntan hacia dietas mixtas que incluyen maíz y otros cultígenos pero con un fuerte énfasis en la caza-recolección (Medina et al. 2014). 
El estudio del proceso de intensificación económica durante el Período Prehispánico Tardío requiere utilizar, entonces, enfoques teóricos y metodológicos lo suficientemente flexibles como para dar cuenta de su dimensión espacial y social. Sólo una perspectiva regional que considere la totalidad del paisaje arqueológico, con poblados estivales, ocupaciones en aleros y sitios de molienda comunitaria dispersos en el espacio serrano, permitirá lograr avances significativos hacia la comprensión de la subsistencia y su impacto en la organización sociopolítica de los grupos tardíos. En consecuencia, la información debería ser complementada con datos provenientes de una mayor gama de sitios, por lo que su excavación adquiere importancia significativa.

En lo que hace específicamente a los estudios zooarqueológicos, se plantea que el NISP refleja con mayor precisión las frecuencias relativas de taxones, siendo la unidad de medida adecuada para medir procesos de intensificación económica en colecciones altamente fragmentadas como las de AT2. El MNI pierde valor analítico ante conjuntos con grandes desvíos estándar en cuanto a la cronología de su formación y su sensibilidad a los criterios agregacionales. También ante la falta de argumentos concretos para sostener la interdependencia entre los especímenes, que debe ser demostrada y no asumida, como es el caso de huesos articulados -excepcionales en contextos arqueológicos en donde se aprovecharon al máximo todos los nutrientes de las carcasas- y dientes insertos en alveolos.

Agradecimientos: Este artículo es el resultado de investigaciones realizadas bajo un subsidio del Consejo Nacional de Investigaciones Científicas y Técnicas (CONICET PIP 112-20080102678) dirigido por el Dr. Eduardo Berberián. Deseo expresar mi agradecimiento a Sebastián Pastor, Juan Pablo Orsi, Bruno Rosignoli y Javier Hanela, quienes aportaron bibliografía y participaron de los trabajos de campo.

\section{Notas}

1 El cálculo de MNI para los huevos de Rheidae se realizó en base a la metodología desarrollada por Bonomo et al. (2008), teniendo en cuenta el peso promedio de especímenes actuales y los arqueológicos.

2 EL RSA fue definido por Broughton (1999: 58) como el número de especímenes identificados por unidad anatómica, normalizado por el número de veces que el elemento está presente en el esqueleto. Esta medida tiene la ventaja de ser simple, replicable y no estar sujeta criterios de agregación (Grayson y Frey 2004).

3 En cuanto al índice de utilidad económica, se utiliza el FUI sin estandarizar establecido por Metcalfe y Jones (1988) para caribú (Rangifer tarantus).

\section{Bibliografía citada}

Barri, F.; M. Martella y J. Navarro

2009 Nest-site habitat by Lesser Rhea (Rhea pennata pennata) in northwestern Patagonia, Argentina. Journal of Ornithology 150: 511-514.

Behrensmeyer, A.

1978 Taphonomic and ecology information from bone weathering. Paleobiology 4: 150-162.

Berberián, E.

1984 Potrero de Garay: Una entidad sociocultural tardía de la región serrana de la Provincia de Córdoba (Rep. Argentina). Comechingonia 4: 71-138. 
Blumenschine, R.; C. Marian. y S. Capaldo

1996 Blind test of inter-analyst correspondence and accuracy in the identification of cut marks, percussion marks, and carnivore tooth mark on bone surface. Journal of Archaeological Science 23: 493-507.

Bonomo, $\mathrm{M}$.

2006 Un acercamiento a la dimensión simbólica de la cultura material en la región pampeana. Relaciones de la Sociedad Argentina de Antropología XXXI: 89-115.

Bonomo, M.; León, D.; Turnes, L. y E. Apolinaire

2008 Nuevas investigaciones sobre la ocupación prehispánica de la costa pampeana en el Holoceno tardío: el sitio arqueológico Claromecó 1 (partido de Tres Arroyos, provincia de Buenos Aires). Intersecciones en Antropología 9: 25-41.

Bovy, K.

2012 Zooarchaeological evidence for sandhill crane (Grus canadensis) breeding in Northwesterns Washington State. Conservation Biology and Applied Zooarchaeology (ed. por S. Wolverton y R.L. Lyman), pp. 23-41. The University of Arizona Press, Tucson.

Broughton, J.

1999 Resource Depression and Intensification During the Late Holocene, San Francisco Bay. Evidence from the Emeryoille Shellmound Vertebrate Fauna. University of California Press, Berkeley y Los Ángeles.

Canals Frau, S.

1953 Las Poblaciones Indígenas de la Argentina. Editorial Sudamericana, Buenos Aires.

Dantas, M. y G. Figueroa

2008 Análisis Tecnológico y Funcional del Registro Cerámico del Valle de Salsacate y Pampas de Altura Adyacentes (Provincia de Córdoba, República Argentina). BAR International Series 1869, Oxford.

De Nigris, $\mathrm{M}$.

2004 El Consumo en Grupos Cazadores Recolectores. Un Ejemplo Zooarqueológico de Patagonia Meridional. Sociedad Argentina de Antropología, Buenos Aires.

Elkin, D.

1995 Volume density of South American camelid skeletal parts. International Journal of Osteoarchaeology 5: 29-37.

Fiore, D. y F. Borella

2010 Geometrías delicadas: Diseños de gravados en cáscaras de huevo de Rheidae recuperados en la costa norte del Golfo de San Matías, Rio Negro. Intersecciones en Antropología 11: 277-293.

González, A.

1943 Arqueología del Yacimiento Indígena de Villa Rumipal (Pcia. de Córdoba). Publicaciones del Instituto de Arqueología, Lingüística y Folklore "Dr. Pablo Cabrera" IV, Universidad Nacional de Córdoba, Córdoba. 
Grayson, D. y C. Frey

2004 Measuring Skeletal Part Representation in Archaeological Faunas. Journal of Taphonomy 2(1): 27-42.

Laguens, A. y M. Bonnin

2009 Sociedades Indígenas de las Sierras Centrales. Arqueología de Córdoba y San Luis. Editorial de la Universidad Nacional de Córdoba, Córdoba.

Luti, R.; M. Bertran de Solís; F. Galera; N. Muller de Ferreira; M. Berzal; M. Nores; M. Herrera y J. Barrera

1979 Vegetación. Geografía Física de la Provincia de Córdoba. En Vazquez, J.; R. Miatelo y M. Roque (eds.), pp. 297-368. Editorial Boldt, Buenos Aires.

Lyman, R.

2008 Quantitative Paleozoology. Cambridge University Press, Cambridge.

Marshall, F. y T. Pilgram

1993 NISP vs. NMI quantification of body-part representation. American Antiquity 58 (2): 261-269.

Medina, $\mathrm{M}$.

2002. Arqueofaunas y tafonomía: la importancia de la caza en contextos formativos tardíos del sector central de Sierras Centrales. Tesis de Licenciatura en Ciencias Antropológicas. Universidad de Buenos Aires, Buenos Aires.

2008 Diversificación Económica y Uso del Espacio en el Tardío Prehispánico del Norte del Valle de Punilla, Pampa de Olaen y Llanura Noroccidental (Córdoba, Argentina). Tesis Doctoral. Facultad de Filosofía y Letras, Universidad de Buenos Aires, Buenos Aires.

2009 Tendencias en el Consumo Prehispánico Tardío de Recursos Faunísticos: Zooarqueología de C.Pun.39 y Puesto La Esquina 1 (Córdoba, Argentina). Archaeofauna 18: 119-136.

2010 Tecnología cerámica, subsistencia y uso del Espacio en el tardío prehispánico de las Sierras de Córdoba (Argentina). Revista Werkén 13: 305-322.

Medina, M.; C. Acosta Hospitaleche, L. Turnes, E. Apolinaire y S. Pastor 2011 Huevos de Rhea pennata en el Holoceno de la Provincia de Córdoba (Argentina): Implicaciones Ambientales, Zoogeográficas y Arqueológicas. Archaeofauna 20: 157-169.

Medina, M.; S. Grill y L. López

2008 Palinología arqueológica: su implicancia en el estudio del prehispánico tardío de las Sierras de Córdoba (Argentina). Intersecciones en Antropología 9: 99-112.

Medina, M. y S. Pastor

2012 Zooarqueología de Sitios Residenciales Tardíos de las Sierras de Córdoba (Argentina, ca. 1100-300 AP): Avances y Perspectivas. Temas de Arqueología, Estudios Tafonómicos y Zooarqueológicos II (ed. por A. Acosta, D. Loponte y L. Mucciolo), pp. 45-66. Instituto Nacional de Antropología y Pensamiento Latinoamericano, Buenos Aires.

Medina, M.; S. Pastor; E. Apolinaire y L. Turnes

2011a Late Holocene subsistence and social integration in Sierras of Córdoba (Argentina): the south-american ostrich eggshells evidence. Journal of Archaeological Science 38: 2071-2078. 
Medina, M.; S. Pastor y E. Berberián

2014 "Es gente fazil de moverse de una parte a otra". Diversidad en las estrategias de subsistencia y movilidad prehispánicas tardías (Sierras de Córdoba, Argentina). Complutum 25(1): 73-88

Medina, M; P. Teta y D. Rivero

2012 Burning damage and small-mammal human consumption in Quebrada del Real 1 (Córdoba, Argentina): an experimental approach. Journal of Archaeological Science 39: 737-743.

Mengoni Goñalons, G.L.

1999 Cazadores de Guanacos de la Estepa Patagónica. Sociedad Argentina de Antropología, Buenos Aires.

2010 Zooarqueología en la práctica. Algunos temas metodológicos. Xama 19: 83-113

Metcalfe, D. y K. Jones

1988 A reconsideration of animal body-part utility indices. American Antiquity 53: 486-504.

Munro, N. y G. Bar Oz

2005 Gazelle bone fat processing in the Levantine Epipaleolithic. Journal of Archaeological Science 32: 223-239.

Outes, F.

1911 Los tiempos prehistóricos y protohistóricos en la provincia de Córdoba. Revista del Museo de La Plata, Tomo VII (Segunda serie, Tomo IV): 261-374.

Pastor, S.

2007 "Juntas y cazaderos". Las actividades grupales y la reproducción de las sociedades prehispánicas de las Sierras Centrales de Argentina. Procesos Sociales Prehispánicos en el Sur Andino. La Vivienda, la Comunidad y el Territorio (ed. por A. Nielsen, M. Rivolta, V. Seldes, M. Vázquez y P. Mercolli), pp. 361-376. Editorial Brujas, Córdoba.

Pastor, S. y E. Berberián

2007 Arqueología del sector central de las Sierras de Córdoba (Argentina). Hacia una definición de los procesos sociales del período prehispánico tardío (900-1573 d.C.). Intersecciones en Antropología 8: 31-47.

Pastor, S. y L. López

2010 Consideraciones sobre la agricultura prehispánica en el sector central de las Sierras de Córdoba. Arqueología de la Agricultura: Casos de Estudio en la Región Andina Argentina (ed. por A. Korstanje y M. Quesada), pp. 208-233. Editorial Magma, Tucumán.

Pastor, S; M. Medina y E. Berberián

2013 Poblados, Casas y Maizales. Arqueología de los Sitios Residenciales Tardíos de las Sierras de Córdoba (ca.1100-300 AP), Argentina Central. Revista Española de Antropología Americana 43: 31-55.

Pastor, S.; M. Medina; A. Recalde; L. López y E. Berberián 2012 Arqueología de la Región Montañosa Central de Argentina. Avances en el Conocimiento de la Historia Prehispánica Tardía. Relaciones de la Sociedad Argentina de Antropología 37: 89-112. 
Potter, J. y S. Ortman

2004 Comunity and cuisine in the prehispanic American Southwest. Identity, Feasting, and the Archaeology of the Greater Southwest (ed. por B. Mills), pp. 173-191. University Press of Colorado, Boulder.

Rivero, D.; M. Medina; A. Recalde y S. Pastor

2010 Variabilidad en la Explotación de Recursos Faunísticos durante el Holoceno en las Sierras de Córdoba (Argentina): Una aproximación Zooarqueológica. Zooarqueología a Principios del Siglo XXI: Aportes Teóricos, Metodológicos y Casos de Estudio (ed. por Gutierrez, M.; M. De Nigris; P. Fernández; M. Giardina; A. Gil; A. Izeta; G. Neme y H. D. Yacobaccio), pp. 321-331. Ediciones El Espinillo, Buenos Aires.

Serrano, A.

1945 Los Comechingones. Serie Aborígenes Argentinos I. Instituto de Arqueología, Lingüística y Folklore de la Universidad Nacional de Córdoba, Córdoba.

Soilbenzon, E.; M. Medina y A. Abba.

2013 Late Holocene Armadillos (Mammalia, Dasypodidae) of the Sierras of Córdoba, Argentina: zooarchaeology, diagnostic characters and their paleozoological relevance. Quaternary International 299: 72-79.

Stiner, M.C.

1994 Honor Among Thieves. A Zooarchaeological Study of Neardental Ecology. Princeton University Press, Princeton.

Stiner, M.C.

2005. The Faunas of Hayonim Cave, Israel. Peabody Museum Press, Cambridge.

Texier, P., G. Porraz; J. Parkington; J. Rigaud; C. Poggenpoel; C. Miller; C. Tribolo; C. Cartwright; A. Coudenneau; R. Klein; T. Steele y C. Verna

2010 A Howiesons Poort tradition of engraving ostrich eggshell containers dated to 60,000 years ago at Diepkloof Rock Shelter. Proceeding of the National Academy of Sciences of the United States of America 107 (14): 6180-6185

Wolverton, S.; L. Nagaoka; J. Densmore y B. Fullerton

2011 White-tailed deer harvest pressure \& within-bone nutrient explotaition during the midto late Holocene in southeast Texas. Before Farming 2008/2 Article 3 http:/ / www.waspress. co.uk/journals/beforefarming/journal_20082/abstracts /index.php 